\title{
Barreras culturales en los adultos mayores con diabetes de comunidades mayas ${ }^{1}$
}

Institución: Universidad Autónoma de Coahuila

\author{
Josué Medina-Fernández ${ }^{2}$ \\ Ana Carrillo-Cervantes ${ }^{3}$ \\ Daniel Sifuentes-Leura ${ }^{4}$
}

\section{ENSAYO}

RESUMEN

La población adulta mayor maya es considerada vulnerable, debido a barreras que influyen en su calidad de vida, tales como su situación económica y las malas relaciones culturales con el profesional de salud, problemática sobre la que versa este ensayo. La deficiencia de políticas públicas enfocadas a mejorar las condiciones de vida del adulto mayor con diabetes, específicamente en zonas indígenas, es un problema que se debe atender en todo el territorio mexicano, un país con una amplia gama de culturas, por ello se requiere crear y evaluar distintos métodos que ayuden a aumentar la calidad de vida de los adultos mayores, mediante la propuesta de proyectos de salud que consideren los pensamientos y aspectos culturales de cada población y que mejoren las relaciones culturales de la población y los profesionales de la salud, máxime considerando que vivir con una enfermedad crónica puede generar cambios en el ámbito familiar, social e individual, por ende, es fundamental reconocer su forma de vivir y aplicar los cuidados transculturales en la población maya.

Palabras clave: adulto-mayor; calidad-de-vida; diabetes-mellitus; poblaciones-vulnerables.

DOI: $10.15517 /$ revenf.v0i36.32826

${ }^{1}$ Fecha de recepción: 23 de marzo del 2018

${ }^{2}$ Licenciado en Enfermería, Maestrando en Enfermería con acentuación en atención al Adulto Mayor, Facultad de Enfermería, Universidad Autónoma de Coahuila. México. Correo electrónico: josuemedinafernandez@,outlook.es

${ }^{3}$ Doctor en Ciencias de la Enfermería, Catedrático e investigador, Facultad de Enfermería, Universidad Autónoma de Coahuila. México. Correo electrónico: alcc73@hotmail.com

${ }^{4}$ Doctor en Ciencias de la Enfermería, Catedrático e Investigador, Facultad de Enfermería, Universidad Autónoma de Coahuila. México. Correo electrónico: sild0320@,hotmail.com 


\section{Cultural barriers in older adults with diabetes from Mayan communities ${ }^{1}$}

Josué Medina-Fernández ${ }^{2}$ Ana Carrillo-Cervantes ${ }^{3}$ Daniel Sifuentes-Leura ${ }^{4}$

Institution: Autonomous University of Coahuila

\section{ESSAY}

\section{ABSTRACT}

The elderly mayan people are considered one of most vulnerable populations in Mexico, having barriers that affect their quality of life, these include the economic situation and the bad cultural relations with health team, so in this essay is written on the problems found in this specific population. Deficiency of focused public policies to improve the living conditions of the elderly with diabetes, specifically in indigenous areas, is a problem to be addressed in all the Mexican territory, since it is a country with a wide range of cultures, for this reason is required to create and evaluate different methods that will help to increase the quality of life of seniors, the proposal of health projects that consider the thoughts and cultural aspects of each population, It is also urgent that these programs improve the cultural relations of the population and health professionals. Living with a chronic illness can generate changes in family, social and individual spheres, therefore, it is important to recognize its way of life and apply the transcultural care in the mayan population.

Keywords: diabetes-mellitus; older-adults; quality-of-life; vulnerable-populations.

DOI: $10.15517 /$ revenf.v0i36.32826

${ }^{1}$ Date of reception: March 23, 2018

Date of acceptance: July 18, 2018

2 Bachelor in Nursing, Master in Nursing with emphasis in attention to the Elderly, School of Nursing, Autonomous University of Coahuila. México. E-mail: josuemedinafernandez@,outlook.es

${ }^{3}$ Doctor of Nursing Sciences, Cathedratical researcher.Faculty of Nursing, Autonomous University of Coahuila. México. E-mail: alcc73@hotmail.com

${ }^{4}$ Doctor in Nursing Sciences, Cathedratical researcher. Faculty of Nursing, Autonomous University of Coahuila. México. E-mail: sild0320@hotmail.com 


\title{
Barreiras culturais em idosos com diabetes de comunidades maias ${ }^{1}$
}

\author{
Josué Medina-Fernández ${ }^{2}$ \\ Ana Carrillo-Cervantes ${ }^{3}$ \\ Daniel Sifuentes-Leura ${ }^{4}$
}

\section{Instituição: Universidade Autônoma de Coahuila}

A população adulta maia mais velha é considerada vulnerável, devido a barreiras que influenciam sua qualidade de vida, como a situação econômica e as relações culturais ruins com o profissional de saúde, problema esse que é objeto deste ensaio. A carência de políticas públicas voltadas à melhoria das condições de vida dos idosos com diabetes, especificamente em áreas indígenas, é um problema que deve ser abordado em todo o território mexicano, um país com um amplo espectro de culturas, motivo pelo qual é necessário criar e avaliar diferentes métodos que ajudam a aumentar a qualidade de vida dos idosos, através da proposição de projetos de saúde que considerem os pensamentos e aspectos culturais de cada população e que melhorem as relações culturais da população e dos profissionais de saúde, especialmente considerando que viver com uma doença crônica pode gerar mudanças na família, social e individual, por isso, é essencial reconhecer seu modo de viver e aplicar o cuidado transcultural na população maia.

Palavras-chave: diabetes-mellitus; idosos; qualidade-de-vida; populações-vulneráveis.

DOI: $10.15517 /$ revenf.v0i36.32826

${ }^{1}$ Data de recepção: 23 de março de 2018

Data de aceitação: 18 de julho de 2018

${ }^{2}$ Bacharel em Enfermagem, Mestre em Enfermagem com ênfase em Atenção ao Idoso, Escola de Enfermagem, Universidade Autônoma de Coahuila. México. Correio eletrônico: josuemedinafernandez@outlook.es

${ }^{3}$ Doutor em Ciências da Enfermagem, Catedrático e pesquisador, Faculdade de Enfermagem, Universidade Autônoma de Coahuila.

México. Correio eletrônico: alcc_73@hotmail.com Autônoma Autônoma

${ }^{4}$ Doutor em Ciências da Enfermagem, Catedrático e pesquisador, Faculdade de Enfermagem, Universidade Autônoma de Coahuila.

México. Correio eletrônico: ild0320@hotmail.com 


\section{INTRODUCCIÓN}

Los adultos mayores (AM) indígenas mayas son considerados una población vulnerable, por encontrarse desprotegida, con antecedentes de problemas económicos y con rezago en los servicios de salud ${ }^{1}$, sin olvidar su condición social y la desigual en salud, lo cual puede influir en la disminución de su calidad de vida relacionada con su enfermedad.

La calidad de vida en salud se define como el impacto sobre el bienestar físico, emocional y social de una enfermedad médica y su tratamiento: representa una percepción subjetiva del impacto de la enfermedad y repercute en la capacidad para enfrentarse a una situación ${ }^{2}$. Dicho concepto se encuentra relacionado con el estado sociodemográfico, la enfermedad, la cultura y la edad ${ }^{3,4}$, por lo que los adultos mayores indígenas mayas con diabetes se encuentran en su gran mayoría con problemas relacionados con factores económicos, del lenguaje y la falta del entendimiento de sus cuidados culturales por parte del personal de salud. Al respecto, se calcula que las poblaciones indígenas mayas cuentan con 575.7 mil personas; de ellas, poco más de 27 mil no hablan español y solo se comunican en maya, por lo que se considera la lengua más hablada en el estado de Yucatán, México, con el $98,1 \% 5$.

Dado lo anterior, y de acuerdo con la estadística de pobreza del Consejo Nacional de Evaluación de la Política de Desarrollo Social (CONEVAL), a nivel nacional Yucatán ocupa el lugar número 15 de pobreza y 11 en pobreza extrema: el 48,5 \% (949,140 de 1, 956,273 yucatecos) corresponde al estado de pobreza y el 11,7 \% (719,440 yucatecos) a pobreza extrema; es decir, el 60,2 \% vive en un rango de pobreza: de este total, el $86.8 \%$ pertenece a una zona rural maya ${ }^{6}$.

Respecto de las limitaciones de los servicios de salud, el 56,8 \% (1,111,806 de personas) no poseen servicios públicos de salud, mientras que la carencia de servicios básicos de vivienda fue de 20,7\%; es decir, 405,622 personas $^{6}$.

De acuerdo con los adultos mayores con diabetes, el $25.6 \%(52,100)$ de las personas de 60 años y más la padecen, de estos solo el 9\% (10,900) se han realizado alguna revisión en los últimos 12 meses $^{7}$.

En cuanto a los datos anteriores, la calidad de vida de los adultos mayores mayas es afectada por cuestiones económicas, del lenguaje y la falta del entendimiento de la cultura, por lo que en este ensayo se aborda los principales problemas que aquejan a la población, así como uno de los puntos prioritarios para mejorar la atención de salud, la transculturalidad, la cual implica el entendimiento de sus cuidados culturales y que este preserve el tratamiento médico con el cuidado cultural. 


\section{DESARROLLO}

\section{Disminución de la calidad de vida en el adulto mayor relacionada con la diabetes}

La calidad de vida es un concepto amplio y variable, ya que puede incluir fenómenos psicológicos, físicos, sociales, económicos y culturales: específicamente, la prevención y protección aplicadas a los fenómenos culturales son la base principal para mantener o mejorar calidad de vida en el adulto mayor.

El supervisar la forma de vida del adulto mayor con diabetes permite conservar sus capacidades funcionales, de modo que se evita riesgos físicos y neurológicos, nefropatías, cardiovasculares, retinopatías, psicológicos, entre otros, factores que es imprescindible considerar en la atención de las poblaciones vulnerables, ya que los adultos mayores pueden hacer prácticas culturales empíricas en su salud sin conocimiento de los efectos que pudieran causarle $^{8}$. La disminución del estado de salud de la población mayor a 60 años en las comunidades indígenas mayas es causada principalmente por las relaciones asimétricas, lo cual provoca como consecuencia la afectación en el impacto del tratamiento 9 .

En relación con lo anterior, las unidades de primer nivel de atención obstaculizan la asistencia sanitaria por la falta de compresión de la cultura, lo cual genera problemas respecto de consensuar los elementos transculturales relacionados con el paciente.

\section{Afección cultural de los servicios de salud}

La escasez de políticas públicas enfocadas en mejorar las condiciones de vida del adulto mayor con diabetes, específicamente en zonas indígenas, es un problema que se debe considerar y abordar en todos los estados de México, considerando lo multicultural, a lo que se suma el nulo alcance que han tenido los servicios públicos de salud lo que impacta en un alza de las complicaciones de la enfermedad ${ }^{10}$. Lo anterior se confirma al contrastarlo con los programas implementados por la Secretaría de Salud en Yucatán, principal promotor de la salud pública estatal, cuyo enfoque se centra en programa aplicados al adulto mayor con deterioro cognitivo, depresión, incontinencia urinaria, caídas y enfermedad de la próstata ${ }^{11}$, mas se detectan deficiencias respecto de prevenir enfermedades crónicas no transmisibles como la diabetes.

Tal como se mencionó, una de las barreras de la atención de las poblaciones indígenas de Yucatán es la relación cultural, a las que se suman las siguientes: la primera corresponde a relaciones asimétricas, cuyas barreras culturales con el personal de salud conciernen a la falta de entendimiento a las creencias de los AM y al desconocimiento de su cultura, lo cual se refleja en un maltrato y desacuerdo con la población maya: un ejemplo de ello es evidente en las medicaciones, las cuales impiden que las personas hagan uso de la medicina tradicional $^{12}$. 
Luego, se encuentran las relaciones interculturales, las cuales se pueden entender como la empatía que muestra el personal de salud en torno a las costumbres y el uso de la medicina tradicional por parte de los AM. En esta barrera el personal de salud muestra respeto, sin embargo, no proporciona un tratamiento basado en la cultura, es decir, lo cual implica cuidados culturales dentro del tratamiento médico que permita la coexistencia de la medicina hegemónica y medicina tradicional con el fin de obtener el control de la glucemia sanguínea ${ }^{9}$.

La última barrera, una de las más importantes para aplicar en las poblaciones, se refiere a las relaciones de los profesionales de salud con servicios holísticos, los cuales apoyan y facilitan en las personas con diabetes la modificación de sus estilos de vida en conjunto con las creencias culturales para conseguir cambios mayores en la salud del paciente ${ }^{12}$.

En cuanto a los retos, uno de los principales consiste en mejorar las políticas en salud que se adapten a la población, apostar por una investigación cuantitativa y cualitativa que ayude a entender mejor el uso de la medicina tradicional, mejorar el alcance de los servicios de salud en la atención del adulto mayor y, sobre todo, adaptar la promoción de la salud a la cultura ${ }^{13}$.

Por todo lo anterior, se considera que las relaciones con el profesional de salud y la falta de políticas públicas representan un factor importante que debilita la calidad de vida del adulto mayor con diabetes, sin dejar de lado que la falta de las adaptaciones de cuidados culturales y la deficiente atención a esta población vulnerable viola sus derechos de acuerdo con la legislación y derechos indígenas de México ${ }^{14}$.

De igual forma, al tomar en cuenta tal problemática, se estará cubriendo varias estrategias del plan de acción de salud 2013-2018, tales como la 1.2, referida a la prevención y control de las enfermedades crónicas; la 1.7 para promover el envejecimiento saludable y la 4.3 que destaca el fortalecimiento de los servicios de promoción y prevención de las poblaciones indígenas y otros grupos vulnerables ${ }^{15}$, a partir de las que la salud de la población adulta mayor con diabetes mantendrá niveles de control metabólico y glicémico adecuados.

\section{Adaptación cultural en el cuidado del adulto mayor}

Una de las posibles estrategias que pueden impactar a corto plazo la mejora de la calidad de vida de los AM indígenas con diabetes sería implementar programas de salud que consideren las cuestiones culturales de la población como coadyuvante para prevenir las complicaciones de la diabetes mas, para lograrlo, es prioritario implementar adaptaciones culturales en la salud a dichos programas.

Dentro de estas adaptaciones se debe respetar su cosmovisión de salud "la salud no solo significa estar bien con uno mismo o con la ausencia de enfermedad, sino que también debe estar bien con los demás, es decir, toma en cuenta la armonía con el mundo y la sociedad"16, así como mantener relaciones transculturales, las cuales permitan a la persona realizar sus cuidados tradicionales, siempre y cuando no se conviertan en una complicación para la enfermedad. 
Por otra parte, se debe sensibilizar al personal de salud para que conozca y respete los cuidados tradicionales, de modo que brinde apoyo para que los adultos mayores mantengan una cultura de autocuidado: así se cumpliría con lo mencionado por la Organización Mundial de la Salud sobre la medicina tradicional 2014-2023, con la que se busca promover y utilizar segura y eficaz la medicina tradicional en los profesionales y en los sistemas de salud ${ }^{13}$.

Respecto de la barrera económica, corresponde tanto al estado de Yucatán como a los profesionales de la salud, la creación de políticas públicas que protejan la salud y la cultura en la comunidad adulta mayor indígena, para plantear la posibilidad de elaborar programas de atención aplicables en todas las poblaciones, máxime en aquellas con problemáticas económicas y de salud en aumento.

\section{CONCLUSIÓN}

Como se ha sabido, el vivir con una enfermedad crónica puede generar cambios en el ámbito familiar, social e individual, por lo que es importante reconocer la forma de vivir y las barreras que mejoran la calidad de vida. Por tal motivo, comprender las creencias, valores y sentimientos de los adultos mayores indígenas es prioritario para los profesionales de enfermería, de modo que planeen e implementen programas que consideren los aspectos tradicionales y culturales de la población a la que estén dirigidos.

Al considerar los aspectos culturales y tradicionales de la comunidad en conjunto con la medicina hegemónica se favorecerá la prevención de algunas complicaciones, dado que se brinda atención específica y adaptada a las poblaciones vulnerables del estado de Yucatán, México.

Conflicto de intereres: Los autores manifiestan no tener conflicto de interés.

\section{REFERENCIAS BIBLIOGRÁFICAS}

1. Medina I, Medina J, Pech M. Desigualdad social en grupos vulnerables. Saltillo, Coahuila; 2016.

2. Herrera P, Guzmán H. Reflexiones sobre calidad de vida, dignidad y envejecimiento. Rev Médica Clínica Las Condes. 2012;23(1):65-76.

3. Sánchez L, González U, Alerm A, Bacallao J. Calidad de vida psíquica y estado de salud física en el adulto mayor. Rev Habanera Ciencias Médicas. 2014;13(2):337-49.

4. Corugedo M, García D, González I, Crespo G, Gónzales G, Calderín M. Calidad de vida en adultos mayores del hogar de ancianos del municipio Cruces. Rev Cuba Med Gen Integr. 2014; 30(2):208-16.

5. INEGI. Resultados Definitivos De La Encuesta Intercensal 2015. Boletín de prensa numero 524; Yucatán; 2015.

6. CONEVAL. Informe de pobreza y evaluación en el estado de Yucatán 2012. Ciudad de México; México; 2012.

7. Instituto Nacional de Salud Pública. Resultados de la diabetes por entidad Federativa. Yucatán. Encuesta Nacional de Salud primera edición. 2012. 113 p. 
8. Chafio P, Gallo G. Calidad de vida del adulto mayor con diabetes mellitus que se atiende en el Centro de Salud José Olaya Chiclayo. [Tesis de Licenciatura]. Repositorio Institucional - USS. Universidad Señor de Sipán; 2016.

9. Medina Fernández J, Medina Fernández I, Candila Celis J, Yam Sosa A. Saberes Y prácticas de una población rural maya con Diabetes Mellitas tipo II sobre las plantas medicinales. RqR Enfermería Comunitaria (Revista de SEAPA). 2018; 6(2): 76-91.

10 . Juárez C, Márquez M, Salgado N, Pelcastre B, Reyes H. La desigualdad en salud de grupos vulnerables de México : adultos mayores, indígenas y migrantes. Rev Panam Salud Pública. 2014;35(4):284-90.

11. Servicios de Salud de Yucatán. Programa de Atención al Envejecimiento. Secretaría de Salud Yucatán. 2016 [actualizado 16 de enero 2015].

12. Quilaqueo D, Torres H. Multiculturalidad e Interculturalidad: Desafíos Epistemológicos de la Escolarización desarrollada en Contextos Indígenas. Alpha (Osorno). 2013; 37(37):285-300.

13. OMS. Estrategia de la OMS sobre medicina tradicional 2014-2023. Organización Mundial de la Salud 2013 [actualizado diciembre 2013].

14. Legislación y Derechos Indígenas en México. México: Camara de diputados y H. Congreso de la Unión: 2010.

15. Gobierno de la República. Programa Sectorial de Salud 2013-2018. 2013 [actualizado noviembre 2014] p. 45-67.

16. Cabrera E. El calendario Maya, su origen y filosofía. Guatemala; 2013. 15-17 p. 